\title{
Jules Romains, " poète de la sociologie »
}

Jules Romains, the "poet of sociology"

\section{Jacques Coenen-Huther}

\section{OpenEdition}

\section{Journals}

Édition électronique

URL : http://journals.openedition.org/ress/3598

DOI : $10.4000 /$ ress.3598

ISBN : 1663-4446

ISSN : 1663-4446

\section{Éditeur}

Librairie Droz

Édition imprimée

Date de publication : 30 novembre 2016

Pagination : 251-276

ISSN : 0048-8046

Référence électronique

Jacques Coenen-Huther, « Jules Romains, "poète de la sociologie » », Revue européenne des sciences sociales [En ligne], 54-2 | 2016, mis en ligne le 30 novembre 2019, consulté le 02 janvier 2020. URL http://journals.openedition.org/ress/3598; DOI : 10.4000/ress.3598 


\title{
JULES ROMAINS, «POĖTE DE LA SOCIOLOGIE »
}

\author{
JACQUES COENEN-HUTHER
}

Université de Genève jacques.coenen-huther@bluewin.ch

\begin{abstract}
Résumé. Jules Romains, homme de lettres, auteur à succès dans la première moitié $\mathrm{du} X \mathrm{x}^{\mathrm{e}}$ siècle, entretint des rapports ambigus avec la sociologie de son époque. Sa doctrine littéraire, l'unanimisme, lui paraissait rejoindre la démarche des sociologues. Cette attitude à l'égard de la sociologie prenait appui sur une tradition française d'émulation entre littérature et sciences sociales. Le manifeste de l'unanimisme qu'il écrivit dans sa jeunesse se présente sous la forme d'un long poème qui attira l'attention de Durkheim. Son œuvre majeure, Les Hommes de bonne volonté, suite romanesque en vingt-sept volumes, se caractérise par un esprit sociologique marqué. L'auteur décrit une foule de personnages insérés dans leurs rapports sociaux respectifs. Les effets de groupe jouent un rôle important dans cette création littéraire. Quant aux rapports entre Jules Romains et les sociologues durkheimiens, particulièrement Halbwachs, ils suggèrent une estime réciproque entre le monde de la littérature et celui de la sociologie.
\end{abstract}

Mots-clés: norme sociale, observation, onde historique, pression sociale, Jules Romains, unanimisme.

\begin{abstract}
Jules Romains, man of letters, was a successful author in the first half of the $x x^{\text {th }}$ century. His relationship to sociology was rather ambiguous. According to him, "unanimism", the literary doctrine he favored, converged with the sociological approach. His attitude toward sociology relied on a French tradition of competition between literature and social sciences. The manifesto of unanimism he wrote when he was young takes the form of a long poem which attracted Durkheim's attention. A distinctive sociological outlook marks out his major work, Les Hommes de bonne volonté, a saga in twenty-seven volumes. The author describes numerous characters inserted in their respective social environment. Group dynamics play an important role in that literary fiction. As for the relations between Jules Romains and Durkheimian sociologists, especially Halbwachs, they point towards a mutual esteem between the world of literary and that of sociology.
\end{abstract}

Keywords: historical wave, observation, Jules Romains, social norm, social pressure, unanimism. 
Jules Romains (1885-1972), homme de lettres - poète, romancier, dramaturge, essayiste - fut un auteur à succès pendant la première moitié du $\mathrm{xx}^{\mathrm{e}}$ siècle; les vingt-sept volumes de son œuvre majeure, Les Hommes de bonne volonté, rédigés entre 1932 et 1947, furent tirés à des milliers d'exemplaires et la parution de chacun d'eux fut un événement littéraire. Depuis la fin des années 1960, ce cycle romanesque s'est néanmoins effacé peu à peu des mémoires au profit d'ouvrages exigeant moins de patience du lecteur. Si leur auteur a accédé au rang de classique de la littérature française, il est actuellement peu lu et les jeunes générations s'en désintéressent. Il est vrai que la mode des romans-fleuves a passé. On ne se bouscule plus non plus pour s'immerger dans la Comédie humaine, les Rougon-Macquart ou Jean-Christophe. De toute l'œuvre de Jules Romains, c'est sans doute le petit roman burlesque Les Copains (19I3), chanté par Georges Brassens et porté à l'écran par Yves Robert, qui a le mieux survécu au passage du temps.

Cet auteur à la fortune changeante a entretenu des rapports ambigus avec la sociologie en voie d'institutionnalisation. Il serait néanmoins faux d’en faire un sociologue qui s'ignore ou un Monsieur Jourdain de la sociologie car il était parfaitement conscient de l'existence de cette discipline en progrès et il avait le sentiment que sa propre doctrine littéraire - l'unanimisme - le conduisait sur une voie parallèle à celle des sociologues. On serait tenté de dire qu'il fut un franc-tireur de la sociologie, voué à une entreprise d'exploration de la vie en société, menée avec d'autres ressources intellectuelles que celles des sociologues attitrés mais qui n'en avait pas moins une inspiration d'ordre sociologique. Il est fréquent qu'on distingue, entre la sociologie et la littérature, une situation de concurrence ou une situation de convergence mais Jules Romains ne se situe ni dans la première configuration ni dans la seconde. Il définit les rapports de l'unanimisme et de la sociologie comme «des rapports de coexistence » dus tout simplement au fait qu'ils sont «les produits d'un même âge de l'humanité » (1933, p. 237). 


\section{L'HÉRITAGE DU XIXE SIÈCLE NATURALISTE}

Il faut ajouter toutefois que dès le XIX siècle s'est établie en France une tradition d'émulation entre littérature et sciences sociales, nourrie par la fascination des sciences de la nature, dont a hérité l'œuvre de Jules Romains bien que celle-ci présente des caractéristiques particulières, comme le signale son auteur (1964, p. I32-I34, p. 136). Ainsi que le fit observer Wolf Lepenies, la situation de compétition, voulue ou non, entre la littérature et la sociologie eut pour toile de fond, à la fin du XviII siècle, l'absence «d'une ligne de démarcation nette entre œuvres littéraires et œuvres scientifiques» (1990 [1985], p. 2). C’est alors que s'amorce un processus de différenciation qui peut certes faire l'objet d’analyses divergentes, comme le souligne opportunément François Chazel (2000, p. 88), mais qui aboutit à l'éloignement progressif de la culture scientifique et de la culture littéraire, consacrant le caractère hybride de la sociologie sur lequel j’ai insisté ailleurs (Coenen-Huther, 2012). La concurrence aboutit parfois, dans le contexte de la III République, à une véritable hostilité à l'égard des sociologues alors que ceux-ci gagnaient peu à peu une reconnaissance officielle. Pour les adversaires du régime républicain tout comme pour les défenseurs de la culture classique, la sociologie - et singulièrement la sociologie d'orientation positiviste que personnifiait Durkheim - était un instrument d'embrigadement intellectuel au service de l'État laïque et anticlérical. En ı9ıo, deux jeunes écrivains, Alfred de Tarde et Henri Massis, publièrent dans L'Opinion, sous le pseudonyme d’Agathon, une série d’articles attaquant violemment les tendances dominantes à la Sorbonne et la place qu'on y faisait à une «pseudo-science» de «nouveaux riches» de la culture: la sociologie. Ces articles furent réunis l'année suivante en une brochure intitulée L’Esprit de la Nouvelle Sorbonne (I9I I). Les auteurs se gaussaient de l'imitation du modèle des sciences naturelles par les sciences humaines et de la crainte de toute intervention de la subjectivité qui en résultait. Abstraction faite du climat politique de l'époque, ces esprits d'orientation littéraire, nourris de culture classique, voyaient bel et bien dans la sociologie une menace pour le patrimoine culturel du pays et pour une véritable compréhension de la réalité sociale. Jules Romains ne partagea jamais une telle animosité mais l'idée que la création littéraire était vouée à n’apporter que des matériaux à la recherche sociologique lui était également étrangère. 
Un état de concurrence de fait fut établi très tôt par des écrivains de renom. Des romanciers comme Balzac et Zola ne cachaient pas leur ambition d'aller au-delà de l'œuvre purement littéraire. Dans sa correspondance, Flaubert note que «la littérature prendra de plus en plus les allures de la science; elle sera surtout exposante, ce qui ne veut pas dire didactique. Il faut faire des tableaux, montrer la nature telle qu'elle est, mais des tableaux complets, peindre le dessus et le dessous » (1980 [1853], p. 298). Le dessus et le dessous: sous les apparences, la structure cachée. En I842, Balzac, présentant la première édition de La Comédie humaine (qu'il avait initialement envisagé de publier sous le titre (Euvres sociales), évoque «l’immensité d'un plan qui embrasse l'histoire et la critique de la Société, l’analyse de ses maux et la discussion de ses principes » (1958, p. 53). Pour caractériser l'ampleur de son projet, il précise qu’il s'agit «de peindre les deux ou trois mille figures saillantes d'une époque, car telle est, en définitive, la somme des types que présente chaque génération et que La Comédie humaine comportera » (ibid., p. 49). Selon l'esprit du temps, il cherche tout d'abord son inspiration du côté des sciences naturelles, indiquant qu'il existera «de tout temps des Espèces sociales comme il y a des Espèces zoologiques» (ibid., p. 37). Paul Bourget, dans un commentaire de son œuvre datant de la fin du siècle, écrit que Balzac «a constaté et mesuré le rapport exact du personnage à son milieu, de même qu’il a constaté et mesuré les attaches de ses différents personnages entre eux; si bien que chacun des individus se trouve constitué séparément dans ses réalités personnelles et sociales, et qu'il en est de chaque famille comme de chaque individu» (I893, p. VI). Balzac anticipe toutefois la notion de social sui generis qui fondera la sociologie durkheimienne et qui, par les effets de groupe, affleure à tout moment dans Les Hommes de bonne volonté: «l'état social, écrit-il, a des hasards que ne se permet pas la Nature, car il est la Nature plus la Société» (ibid., p. 37 ; souligné par nous). Il insiste ainsi, rejoignant la philosophie d’Émile Boutroux (1874), sur l'élément de contingence propre aux entreprises humaines.

De façon inattendue, les classiques du marxisme firent état à plusieurs reprises d'une forme de convergence entre La Comédie humaine et leurs propres analyses. Marx et Engels furent des lecteurs attentifs - et admiratifs - des romans de Balzac et échangèrent du courrier au sujet de ces lectures (Marx 
et Engels, 1954, p. 320-32I). Dans une lettre d’avril ı888 à la femme de lettres social-démocrate Margaret Harkness, Engels déclare que Balzac

nous donne dans La Comédie humaine l'histoire la plus merveilleusement réaliste de la société française, en décrivant sous forme d'une chronique des mœurs, presque d'année en année, de 1816 à I848, la pression de plus en plus forte que la bourgeoisie ascendante a exercée sur la noblesse qui s'était reconstituée après I815. [...]J’y ai plus appris même en ce qui concerne les détails économiques (par exemple la redistribution de la propriété réelle et personnelle après la révolution), que dans tous les livres des historiens, économistes, statisticiens professionnels de l'époque, pris ensemble (ibid., p. 318).

Marx, dans Le Capital, est encore plus spécifique :

Dans une société dominée par la production capitaliste le producteur non capitaliste lui-même est dominé par les conceptions capitalistes. Dans son dernier roman Les Paysans, Balzac, remarquable par sa compréhension profonde des rapports réels, décrit avec une très grande justesse comment le petit paysan, afin de conserver la bienveillance de son usurier, exécute gratuitement pour lui toutes sortes de travaux, sans se figurer par là lui faire des cadeaux, parce que son propre travail ne lui impose pas de dépense proprement dite. L'usurier, de son côté, fait ainsi d'une pierre deux coups. Il s'épargne le versement d'un salaire et enveloppe de plus en plus étroitement dans les filets de l'usure le paysan ruiné progressivement parce qu'il délaisse le travail sur son propre champ (ibid., p. 320-32I).

Zola, pour sa part, entreprend le cycle des Rougon-Macquart, histoire d'une famille sous le Second Empire, qu'on peut concevoir comme l'équivalent pour cette période de ce qu’avait été La Comédie humaine pour la Monarchie de Juillet. Ses Carnets d'enquête préparatoires à l'œuvre révèlent un romancier qui pousse le souci de la documentation préalable jusqu'à la pratique d'une véritable observation participante avant la lettre. Dans son introduction à l'édition «Terre humaine» des Carnets, Jean Malaurie évoque Zola qui «tel un ethnographe contemporain, va sur le terrain, interviewe les banquiers, monte sur une locomotive, descend dans la mine, parcourt les rayons de grands magasins, $[. .$.$] interroge les$ vendeurs et les clients » (1986, p. II). Mais au-delà de ce «travail de terrain » qui pourrait ne refléter que le souci d'une documentation aussi riche que possible, c'est une conception quasi sociologique de la vie sociale qui émerge comme, 
plus tard, chez Jules Romains. Zola tient à peindre non seulement des individus mais aussi des groupes, des ensembles. Il perçoit de manière très lucide l'interpénétration de l'individuel et du collectif: «notre grande étude est là, dans le travail réciproque de la société sur l'individu et de l'individu sur la société »; il s'agit de «montrer l'homme vivant dans le milieu social qu'il a produit luimême, qu'il modifie tous les jours, et au sein duquel il éprouve à son tour une transformation continue » (I890, p. 19). Le rôle du romancier est à ses yeux celui d'un «moraliste expérimentateur» dégageant «le déterminisme des phénomènes sociaux pour qu'on puisse un jour, dans une perspective comtienne, dominer et diriger ces phénomènes» (ibid. p. 29). Ainsi, le roman lui paraît devenu «une enquête générale sur la nature et sur l'homme» (ibid. p. 36-37). Dès lors, il n'hésite pas à écrire que «nous faisons de la sociologie pratique» (ibid., p. 24) car le roman expérimental se situe à l’aboutissement d'une chaîne de causalité s'échelonnant «de la chimie à la physiologie, puis de la physiologie à l'anthropologie et à la sociologie» (ibid., p. 2). Pour Flaubert,

quand on aura, pendant quelque temps, traité l'âme humaine avec l'impartialité que l'on met dans les sciences physiques à étudier la matière, on aura fait un pas immense. C'est le seul moyen qu'a l'humanité de se mettre un peu au-dessus d'elle-même. Elle se considérera alors franchement, purement, dans le miroir de ses œuvres (1980 [1853], p. 451).

Et bien, ajoute-t-il, «je crois cela faisable. C'est peut-être, comme pour les mathématiques, rien qu’une méthode à trouver» (ibid.).

Une convergence entre la sociologie et la littérature est souvent perçue de manière réductrice comme une forme de subordination de la seconde à la première, ce que Jules Romains s'efforça d'éviter lorsqu'il en fut à la phase de théorisation de l'unanimisme. La réalité est en effet plus complexe.

Comme l'écrit Pierre Lassave :

en ses divers genres, la littérature est pour le sociologue (comme pour l'historien et plus récemment pour l'ethnologue) une source inépuisable d'informations, de schémas, de procédés expressifs et de systèmes d'interprétation qui savèrent d’autant plus pertinents que le raisonnement, l'enquête et le chiffre peinent à rendre compte de l'ambivalence des sentiments ou de l'incongruité des situations et à user d'empathie avec des milieux sociaux inaccessibles ou disparus (2002, p. 33). 
En d'autres termes, les œuvres littéraires offrent certes une documentation souvent précieuse et jouent à cet égard un rôle auxiliaire mais leur fonction ne se limite nullement à l'apport de documents. Elles contribuent également à donner sens aux données recueillies car elles constituent une source d'interprétations possibles dont le chercheur tire profit autant que du contact direct avec la réalité. Loin de n'être qu'un informateur, au sens ethnologique du terme, le romancier devient alors un médiateur entre le sociologue et la réalité sociale. Pour le meilleur et pour le pire, osera-t-on dire, car son rôle est positif quand il contribue, comme c'est souvent le cas, à affiner le regard sociologique mais il est incontestablement négatif lorsque le sociologue néglige de manière imprudente le caractère inévitablement sélectif d’une documentation passée au filtre de la sensibilité de l'artiste et procède à des généralisations peu défendables.

\section{ENTRE POÉSIE ET SOCIOLOGIE: L'UNANIMISME}

Au début du $\mathrm{Xx}^{\mathrm{e}}$ siècle, on note un nouvel essor du naturalisme littéraire sous la forme de l'unanimisme qui conserve l'ambition de peindre non seulement des individus et des groupes mais des ensembles matériels - une rue, une place, une ville - et d'en faire des personnages collectifs à la manière dont Zola traita le grand magasin dans Au bonheur des dames. Parmi les écrivains unanimistes de l'époque, on compte Georges Chennevière, qui sera le co-auteur avec Jules Romains d'un Petit traité de versification en 1923, et les poètes-typographes du groupe dit de l’Abbaye, ainsi nommé en raison de l'expérience communautaire tentée à Créteil en 1906 - en référence à l'Abbaye de Thélème chère à Rabelais - par des écrivains et artistes, à l'initiative de Charles Vildrac et de Georges Duhamel qui, plus tard, évoquera cette aventure dans Le Désert de Bièvres, tome V de la Chronique des Pasquiers. En 1908, le jeune Jules Romains - il avait vingt-trois ans - publia un long poème épique, La Vie unanime, édité aux éditions de l’Abbaye, qui bénéficia d’une abondante critique, souvent élogieuse. Dans le premier numéro de la NRF, André Gide, s'en tenant aux qualités littéraires, écrivit: «Je tiens ce livre de débutant pour un des plus remarquables et significatifs que nous ait donnés la génération qui s’élève ». Cette publication, que sa structure empêchait de confondre avec un simple recueil de poèmes, sera considérée comme une sorte de manifeste de l'unanimisme et 
annoncera la suite de l'œuvre de Jules Romains, lui donnant une incontestable coloration sociologique pour qui sait lire en filigrane:

Les frères, les cousins et les petits enfants / Sont venus chez l'aïeul pour faire la veillée; / On cause par instants. Les voix ne sonnent plus / Comme aux jours de marché quand on vend une vache / Et ne se gonflent pas en ampoules remplies / De jurons. Les muscles et les mots se détendent. / Il se fait des accords de syllabes chantantes / Entre phrases d'enfants et phrases de vieillards. / Et peu à peu les voix ne sont plus à personne; / La veillée cause. (1983 [1908], p. 186).

Des gens arrivent pour prier dans leur église. / Malgré qu'il se fatigue et commence à branler, / Le monument sait faire encore une assemblée / Avec les hommes que déversent les chemins. / Pendant qu'ils entrent sous le porche, il les tamise; / Il ôte doucement à chacun des pensées / Qu'il ne pourrait pas fondre aussi bien que les autres / Et les remplace par d'anciennes qu'ont laissées / Ceux qui venaient s'asseoir aux messes de jadis. (ibid., p. 74).

La ville vers le nord est bossue de faubourgs; / Elle s'épanouit, elle gonfle, elle pousse; / Elle s'exalte en excroissances palpitantes / Qui sont des embryons et des printemps de ville, / Où pullulent des hommes pauvres et musclés. / Hâtivement, ils se reproduisent et meurent. / Il y a plus d'enfants que de vieillards, au nord, / Il y a plus d'usines que d'églises. (ibid., p. 152).

Dans le prolongement du naturalisme, l'unanimisme prescrivait à l'écrivain de ne décrire que des personnages insérés dans leurs rapports sociaux, mettant ainsi l'accent sur la vie collective au sein des groupes humains. Jules Romains peine de toute évidence à offrir une définition claire de l'unanimisme qu'il qualifie d'«antimonadisme» (1933, p. 23I). Il n’y voit ni une doctrine littéraire ni un système philosophique mais plutôt une «attitude générale» face à la vie ou encore un «style de l'esprit». Pour mieux se faire comprendre, il procède par analogie, évoquant d’autres termes exprimant également selon lui des dispositions d'esprit très générales comme, par exemple, le rationalisme (ibid., p. 222-225). L’attitude unanimiste lui paraît prendre sa source dans une expérience singulière qui pourrait être comparée à l'expérience religieuse. On pourrait en parler comme d'un sentiment de communion - fût-il fugitif - avec une foule ou avec un groupe constitué. Dans son propre cas, ce fut «l'illumination de la rue d’Amsterdam» qu'il éprouva alors qu’il était encore lycéen et dont témoignèrent plusieurs de ses amis. 
À cette heure, la foule était dense; les omnibus, les voitures à chevaux passaient en brinquebalant sur les pavés de la chaussée. Jules Romains, comme à l'accoutumée, marchait à côté de son ami. Et soudain, que se passa-t-il en lui? Quel mécanisme intérieur fut déclenché? Cette rue, ces lumières, ces gens qu’il croisait sur le trottoir montant, il ne les avait jamais vus de cet oeil [...] cette solitude qui d'ordinaire pesait sur ses épaules s'était comme envolée. Et un autre sentiment l'envahissait [...] : celui d’appartenir à cette foule, d'en être un élément inséparable, de vivre avec elle une minute unique et irremplaçable (Bourin, 196I, p. I07).

Les effets de groupe ou de foule jouant un rôle important dans la genèse des comportements sont fréquemment mis en scène dans Les Hommes de bonne volonté. Lorsque l'influence diffuse de la foule environnante transforme un état de conscience individuel, comme dans «la rue d’Amsterdam», l'évocation de la scène fait inévitablement penser aux niveaux de la vie consciente de Bergson (Bourin, ibid., p. I08). Dans le cas contraire - quand l'impact du collectif s'actualise en action -, c'est l'influence de Gustave Le Bon qui semble perceptible. Pour ce dernier, le seul fait que des individus soient «transformés en foule les dote d'une sorte d'âme collective. Cette âme les fait sentir, penser et agir d'une façon tout à fait différente de celle dont sentirait, penserait et agirait chacun d'eux isolément» (1895, p. I5). À la limite, il y a fusion des consciences individuelles et le groupe devient un acteur collectif, comme c'est le cas dans Les Copains où la cohésion du groupe est favorisée par la participation à une entreprise commune - en l'occurrence, un canular - stimulant une amitié lyrique et à une gaieté rabelaisienne atteignant au rire vengeur. Selon la harangue de fin de repas qui clôture le récit, «vous possédez encore depuis ce soir, l’Unité Suprême. Elle s'est constituée lentement. J'en ai suivi la gestation. Ce soir vous êtes un dieu unique en sept personnes, inutile de le cacher!» (1976 [1922], p. I53).

L'unanimisme, lorsqu'on tente d'en faire la théorie, implique deux postulats qu'on pourrait aussi bien qualifier d'articles de foi. Tout d'abord, le postulat de l'unanime, c'est-à-dire la croyance en l'existence d'une réalité collective de nature spirituelle. Ensuite, le postulat de la continuité psychique, c'est-à-dire l'acceptation de la possibilité pour l'individu d'entrer en contact direct, intuitif, avec la collectivité. Cette réalité collective dont l'individu peut prendre conscience, c'est ce que Jules Romains appelle l'unanime. Il ne s'agit donc pas - comme le choix 
du terme pourrait le faire penser - d'un accord unanime des esprits, mais bien d'une réceptivité particulière à l'influence du collectif et d'une absorption de l'individu dans le groupe. On pourrait y voir une forme d'intersubjectivité au sens où l'entend François Chazel lorsqu'il parle d’ajustements cognitifs se déroulant «sur fond d’interaction» et s'opérant «d'autant plus aisément que l'interaction est plus forte entre membres d'un même réseau, voire d'une même collectivité » (1997, p. 205). Tel que Jules Romains l'expliquait, l'unanimisme correspondait chez lui à une triple motivation profonde: tout d'abord «le désir d'explorer et d'exprimer une réalité très insuffisamment connue; ensuite, l'ambition de substituer aux anciens dogmes «un nouveau lien de nature religieuse »; enfin, le besoin de s'évader «des marécages de l'âme individuelle» (1964, p. 40-4I). Un roman d'inspiration unanimiste, écrit-il, peut être amené «à prendre à l'égard de la réalité sociale, une attitude objective qui ne sera pas sans parenté avec celle du sociologue» (1933, p. 238). Son propos s'appuie sur une comparaison avec les romans dits psychologiques. Comme on a pu dire qu'un romancier est fin psychologue, «on dira, si l'on veut, que le romancier est sociologue, ou bon sociologue» (ibid., p. 238). À la parution de La Vie unanime, l'ouvrage reçut un accueil favorable au-delà des cercles de poètes et de critiques littéraires. Des philosophes et des sociologues manifestèrent un intérêt inhabituel, ce qui semble indiquer que l'éloignement des deux cultures n'était pas encore complètement achevé à l'époque. Jules Romains, nous dit-on, adressa des exemplaires du livre à Durkheim et à Le Bon. On peut supposer qu'il s'agissait des «hommages d'auteur» habituels et rien ne permet de penser qu'il n'y eut pas d'autres destinataires de tels envois. L'auteur n'a vraisemblablement pas négligé à cette occasion Bergson, le philosophe des «Données immédiates de la conscience». Celui-ci en tout cas s'est dit «frappé par ce poème d’une inspiration si originale, tout imprégné d’idées panthéistes» (cité par Rony, 1993, p. 142). Quant à Le Bon, il n’hésita pas à déclarer que «les poètes voient fort bien des ensembles que nous analysons fort mal» (ibid., p. I42). Quoi qu’il en soit, avoir songé à placer Durkheim sur la liste des «hommages » était sans doute l'indice d'un sentiment de connivence avec la pensée sociologique, conçue au-delà de tout aspect technique comme une tournure d'esprit ou, 
mieux, comme un certain regard porté sur la réalité sociale. Comme le souligne Monique Hirschhorn, «la sociologie était trop à la mode, l'école durkheimienne trop au centre des polémiques » (1997, p. 75) pour qu'elle n’ait pas attiré l'attention de Jules Romains, normalien, familier des cénacles littéraires.

Mais il se fait que Durkheim prit la peine d'écrire au jeune auteur pour le remercier. Dans sa lettre écrite alors qu'il entreprenait la rédaction des Formes élémentaires de la vie religieuse, Durkheim déclarait avoir depuis longtemps «l'impression qu'il y a dans ces mystères de la vie sociale de quoi inspirer un poète; rien de plus naturel d'ailleurs, ajoutait-il, si, comme je le crois, ce sont ces mystères qui sont tout le réel de ce que les religions essayent de traduire symboliquement». Après avoir évoqué des personnages de la vie courante, il suggérait que la connaissance ordinaire n'en perçoit que «leurs gestes extérieurs [...] dont les effets superficiels sont seuls sensibles » alors que, écrivit-il, s’adressant à Jules Romains,

des forces y jouent, des influences s'y entrecroisent que l'œil n’aperçoit pas. De tout cela, vous donnez fortement le sentiment, beaucoup mieux que nous autres, sociologues, avec nos formules abstraites et exiguës. Si bien que votre œuvre, en dehors de sa valeur poétique a un véritable intérêt scientifique (cité par Rony, 1993, p. I42).

Dans ce courrier, il faut évidemment faire la part de la courtoisie et de la bienveillance d'un notable de la Sorbonne parvenu au faîte de sa carrière à l'égard d'un auteur débutant. Il semble qu'il n’y eut plus d’autre échange entre les deux hommes. Le contact avec les durkheimiens, bien qu'il restât ténu, ne s'interrompit cependant pas à la mort de Durkheim. Dans son Bilan de la sociologie française contemporaine, Célestin Bouglé (1938 [1935]), ce durkheimien ambivalent et quelque peu dissident, suggère habilement une estime réciproque entre le monde de la littérature et celui de la sociologie lorsque, citant Durkheim, il indique que «Jules Romains, poète de la sociologie»- expression reprise dans le titre de cet article - a qualifié ce dernier de «Descartes de l'unanimisme» (ibid., p. I7).

Maurice Halbwachs qui se situait dans la mouvance durkheimienne mais qui n'en fut pas moins influencé par Bergson, attentif aux données de l'intuition, aimait illustrer ses cours par des références à Jules Romains, n’ayant aucune peine à traiter ses textes comme des sources de documentation crédibles. Pour distinguer les formes de sociabilité en milieu villageois et en milieu urbain, 
il indique que «les familles sont plus rapprochées l'une de l'autre, dans le cadre du village, fondues parfois en une chaude intimité presque animale : tel Cromedeyre-le-Vieil de Jules Romains » (ibid., p. I6), ce terroir dont on a pu dire qu'il constituait pour Jules Romains une «véritable déification de sa province » (Rony, 1993, p. 15). Après avoir décrit les habitudes et les styles de vie des différentes classes sociales, Halbwachs poursuit:

un écrivain contemporain qui est en même temps un bon observateur des choses urbaines, Jules Romains, dans un de ses derniers ouvrages, dans un volume de la série Les Hommes de bonne volonté, a retrouvé de façon assez pittoresque ces contrastes et ces nuances dans l'aspect même de certaines rues et de certains quartiers. Il y a, dit-il, dans les grandes villes, des quartiers qui évoquent des idées différentes, quant à la richesse de ceux qui y habitent. Il y a ceux où l'on sent la richesse acquise depuis longtemps [...] à distance de tous les établissements où l'on travaille. Il y a des quartiers où il y a aussi de la richesse, mais une richesse récente [...] dont les racines sont visibles. On habite là à proximité des quartiers d'affaires [...] Et puis, il y a enfin ceux où la richesse est tout à fait en formation [...] on voit des maisons appartenant à des gens riches mais qui habitent en quelque sorte au milieu ou à proximité du champ dans lequel ils travaillent (2008, p. 71-72).

Selon le raisonnement proposé par Halbwachs, l'étude des classes sociales, de leurs différences et de leur hiérarchie, doit se faire selon un double point de vue : celui de la profession et celui des revenus. Mais dans ce cadre méthodologique général, les descriptions des différents quartiers par Jules Romains, fondées essentiellement sur des signes extérieurs de richesse ou d'activité, permettent de supputer le genre de vie de ceux qui y vivent. La richesse établie de longue date se retire dans «des maisons silencieuses et des parcs». La richesse d'origine plus récente coexiste avec «des magasins, peut-être des bureaux, des maisons dans lesquelles on imagine que doit siéger de temps en temps un conseil d'administration ». La richesse encore en mouvement donne lieu à des bureaux «bourdonnants et fiévreux » ou des grands magasins «dans lesquels l'activité est à son plein» (ibid., p. 71-72). 
Halbwachs, attaché à la notion de milieu comme variable indépendante, fut séduit par le parti que tirait Jules Romains de sa connaissance du monde de la grande ville, acquise par observation directe au cours de longues promenades dans les rues de Paris. Son intérêt allait toutefois également aux manifestations de vie collective que l'auteur mettait en évidence. «Dans la pièce de Jules Romains intitulée Le Docteur Knock, un médecin de campagne qui a réussi à persuader le plus grand nombre des habitants qu'ils étaient malades et devaient se soigner, dit à un confrère, en lui montrant par la fenêtre le village, les maisons, les fermes : "La médecine maintenant vit ici avec intensité" ». Et Halbwachs d'ajouter que toute la vie politique d’un pays est faite de périodes d’intensité variable, suscitées ou entretenues par «les parlementaires, les états-majors des partis, les journalistes» qui pourraient dire eux aussi à certains moments que «la politique maintenant vit ici avec intensité» (1964, p. 233). Dans le cadre de ses études de morphologie sociale, Halbwachs ne négligeait pas les groupements qui par nature ne peuvent être qu'éphémères : Jules Romains, écrit-il,

nous a décrit sociologiquement ces rassemblements éphémères qu'on peut observer dans les rues de nos cités, cortèges à l'occasion d'un enterrement, d'une manifestation, attroupements de badauds, queues qui se forment à l'entrée d'un théâtre, groupes qui stationnent dans l'attente d'un autobus: en de telles formations collectives prêtes à se dissoudre, le corps est apparent, c'est l'âme qui est flottante, peu consistante (1938, p. 55 ; souligné par nous).

Il est intéressant de constater qu'Halbwachs qualifie la description de Jules Romains de sociologique mais utilise pour s'y référer des concepts - le corps, l'âme - plutôt en consonance avec l'esprit unanimiste.

Le problème d'une méthode ou d'un cadre d'analyse se posait-il pour Jules Romains comme il s'est posé pour Balzac et pour Zola? Il semble que ce ne fut pas le cas; Alain Lancelot (1990a) et Monique Hirschhorn (1997) poussent l'analyse plus loin qu'il n'est nécessaire dans cette voie. La pensée et le style narratif de Jules Romains étaient imprégnés d'esprit sociologique, c'est-àdire de la propension à décrire la vie sociale comme un écheveau de rapports sociaux interdépendants et complémentaires. Mais ce regard implicitement sociologique se situe au-delà des divergences d'écoles qui ne le concernaient 
pas, même si certains personnages des Hommes de bonne volonté apparaissent selon les cas comme des créatures de Durkheim ou de Weber. Jules Romains, pour les besoins de sa construction romanesque, était tour à tour individualiste ou holiste, adepte d'une démarche positive ou d'une approche compréhensive, orienté vers les réalités ou vers les représentations. Évoquer à son sujet une «lecture boudonienne » ou une «lecture structuraliste » a quelque chose d'incongru. Son œuvre tout entière suggère la possibilité d'une synthèse entre points de vue divers qui se combinent ou se chevauchent; ce sont ses personnages qui accordent la préférence à l'un ou l'autre d’entre eux.

\section{UN LIVRE EN CHANTIER}

Une quinzaine d’années après la parution de La Vie unanime, Jules Romains entreprit la mise en chantier de la fresque unanimiste qui allait devenir la pièce maîtresse de son œuvre: Les Hommes de bonne volonté (1958 [1932-1947]). Sa préparation fut organisée de façon minutieuse et Jules Romains en parle à la manière d'un bon artisan, conscient de ses moyens et de leurs limites; il sagissait, écrivitil en paraphrasant presque Balzac, de «tracer de notre époque une image aussi ressemblante que le permettaient et les ressources actuelles de l'art littéraire, et l'usage que j’avais acquis de ces ressources » (ibid., t. IV, p. I 127). Son souci était de «chercher un mode de composition qui nous permît d'échapper à nos habitudes de vision "centrée sur l'individu”» (ibid., t.I, p. 9). Quarante ans plus tard, en réponse à la question «ai-je fait ce que j’ai voulu? », il décrivit en détail les différentes étapes de ce travail préparatoire (Romains, 1964). Selon sa propre narration, même s'il lui arriva sans doute d'accentuer après coup le caractère systématique de cette mise en chantier, c'est dans un esprit proprement sociologique qu'il procéda, distinguant les variables pertinentes à prendre en considération; on peut imaginer un sociologue sensible à la dimension historique des rapports sociaux, préparant une enquête de terrain, qui ne s'y serait pas pris très différemment. Ceci donne à penser que s'il y eut une influence directe des travaux de Durkheim sur la conception du livre, c'est au cours de cette période de préparation qu'elle se manifesta, alors que Jules Romains approchait de la quarantaine, et non dans sa prime jeunesse comme l'ont suggéré certains commentateurs. 
L'idée était de créer un roman de grande ampleur «qui eût été comme une synthèse unanimiste de notre époque », le but étant d'«exprimer aussi fidèlement et largement que possible une phase de notre vie collective» (ibid. p. I04-I06). Les multiples personnages devraient donc leur existence ou leur apparition sur scène à la trame d'ensemble de leur environnement historique et social. Aucun d'entre eux - personnage de fiction ou figure historique - ne devrait apparaître comme l'élément central de toute la composition. Il fallait éviter aussi de faire sortir des coulisses le romancier, deus ex machina de l'entreprise. Comme le fit observer André Maurois, ce sont les deux personnages principaux de l'œuvre, Jallez et Jerphanion, qui préservent l'auteur de cette position inconfortable pour un écrivain unanimiste en représentant «l'un son côté lyrique, l'autre son côté réaliste» (1965, p. 286), dialoguant à de multiples reprises pour exprimer leurs réactions et leurs sentiments sur les différents événements qu'ils traversent. La première question qui se posa - question sociologiquement et anthropologiquement pertinente - fut celle de la «distance préférable» entre l'écrivain et la société qu'il souhaite décrire. Le choix se porta sur une époque à l'égard de laquelle le romancier peut prendre «une attitude ambivalente», c'est-à-dire «une époque qui, d’une part, soit déjà du passé », autorisant un certain détachement, mais qui, d’autre part, relève du passé de l'écrivain lui-même, lui parlant «le langage immédiat de l'âme», évoquant souvenirs et regrets sans exiger «le détour obligatoire par le document» (ibid., p. I07-I08). C'est finalement le quart de siècle s'écoulant de 1908 à 1933 qui fut retenu. Comme le note Michel Winock, l'auteur visait «une histoire totale, décrivant les diverses couches sociales, à travers plusieurs dizaines de destinées qui, malgré leur discontinuité, leur hétérogénéité, se trouvent réunies dans un processus global dont les protagonistes ne sont pas conscients » (1997, p. 310). Dans une décennie de tensions entre la France et l'Allemagne, marquée notamment par l'incident d’Agadir, l'année 1908 annonçait la fin du «monde d'hier » et préfigurait la catastrophe européenne de I9I4; l'année 1933, assombrie par l’arrivée au pouvoir d’Hitler, signalait la fin de l'équilibre précaire ayant préservé la paix en Europe dans l'entre-deux-guerres et faisait pressentir une nouvelle catastrophe, impliquant des formes «perverses » d’unanimisme totalitaire (Romains, 1964, p. 39). 
Ce «gabarit de vingt-cinq ans » faisait sens, historiquement et sociologiquement, mais participait également des contraintes de la construction romanesque (ibid., p. I Io). Pour les besoins de la création littéraire, il s'imposait de le structurer mais la structure imaginée devait offrir une analogie avec le changement social. C'est ici qu'intervint la notion d'onde historique dont Jules Romains admit sans difficulté qu’elle était purement empirique. Il lui semblait qu'à l'époque moderne, l'histoire du monde occidental pouvait être divisée en périodes d'une «ampleur moyenne de vingt-cinq ans ». Dès lors, la métaphore de l'onde s'avérait fructueuse pour figurer l'enchaînement des événements tout comme leur répétition. On pouvait imaginer «une suite d'ondes» comportant «un léger chevauchement d'une onde sur la suivante», analogue au mouvement de la marée montante sur une plage, chaque onde semblant reproduire en partie la précédente. Cette comparaison permettait d'établir un rapport entre l'ampleur moyenne de l'onde «et la durée moyenne d'effervescence, de pleine efficacité d'une génération » (ibid., p. Io9). Des notions complémentaires s'associaient dans l'esprit de l'écrivain et venaient préciser le mouvement de l'histoire. Octobre 1908, début du récit, serait «le pied de l'onde». La charpente de l'œuvre étant posée, le flux devait s’élever en six années de récit vers «la première arête d'août I9I4» pour atteindre, deux ans plus tard «la crête de l'onde: Verdun ». Ensuite, le reflux se ferait par paliers, pendant que se rassembleraient les forces qui créeraient l'onde suivante (ibid., p. I Io-I I3). Cette image du flux et du reflux évoque tour à tour les constantes mises en évidence par la sociologie formelle et une analyse nuancée de l'évolution sociale.

La structure générale du roman fixait ainsi des limites et des repères à la fresque unanimiste. Il restait à la garnir des personnages qui allaient lui donner vie. Il ne fut pas question d'effectuer un véritable échantillonnage «de toutes les professions et conditions » mais un effort fut entrepris pour accorder aux principaux groupes socioprofessionnels «l'importance qui est la leur» tout en se plaçant «au point de vue des intéressés » (ibid., p. 124). L’attitude du romancier reposait donc sur une combinaison d'observation objective de la totalité sociale et d’approche compréhensive de l'univers mental de chaque individu. On pourrait y voir l'anticipation d'une synthèse entre l'approche durkheimienne 
et l'approche weberienne. Les personnages du roman étaient créés pour être représentatifs des milieux dont la société de l'époque paraissait constituée mais ils n'en conservaient pas moins leur idiosyncrasie particulière: on ne pouvait donc les réduire à des types-idéaux ou à des «acteurs banalisés » comme ce pût être le cas chez un sociologue qui n'eût pas les préoccupations du romancier. Aux yeux de Jules Romains, les principaux milieux sociaux qui devaient être insérés dans le tableau d'ensemble étaient le monde intellectuel et artistique, le monde des grandes affaires, le monde ouvrier, le monde politique, le milieu militaire, l’Église, l'enseignement laïque et républicain, l’aristocratie de naissance, la bourgeoisie provinciale et la paysannerie; ces différents milieux correspondaient à des domaines d'activité mais aussi à des attitudes face à la vie (Romains, 1964, p. 135-I36), autrement dit à des conceptions du monde.

En se fondant sur les catégories adoptées par Jules Romains, Alain Lancelot a analysé le «positionnement social des cinquante personnages principaux du livre» (1990b, p. 174-175). Il en conclut que les deux tiers des personnages recensés relèvent «des couches dirigeantes ou influentes», le monde intellectuel et artistique y étant surreprésenté par rapport à d'autres catégories. Les couches moyennes, en marge de la bourgeoisie ou plus proches de la classe ouvrière, représentent environ un cinquième du total. Le monde ouvrier et la paysannerie constituent $12 \%$ de l'ensemble. Ce recensement rapide suggère, comme le note Lancelot, «une pyramide sociale inversée » et une attention particulière pour «le haut de l'échelle sociale» (ibid., p. I75) résultant sans doute d’un intérêt marqué pour les coulisses de l'action politique. Il faut néanmoins faire observer que les différents personnages inclus dans le calcul n'ont pas le même poids dans le roman si l'on se base sur le volume de texte qui leur est consacré et sur la bienveillance qu'ils y rencontrent.

Quoi qu'il en soit, le désir de l'auteur d'explorer des milieux sociaux très variés est incontestable. La fresque des Hommes de bonne volonté n'est pas centrée sur l'histoire de l'amitié entre Jallez et Jerphanion même si celle-ci ressurgit au gré des événements. Les deux héros sont les témoins, plus ou moins engagés selon les cas, de péripéties qui affectent l'Europe entière. Le pied de l'onde historique annonce la première phase de ce qui fut pour Jules Romains une «guerre civile européenne», expression qui 
sera largement reprise par les historiens depuis lors. La fin de l'onde - 1933- nous montre hélas que chaque vague reproduit le mouvement de la précédente, ce qui se traduit par «les mêmes enthousiasmes, les mêmes folies, les mêmes déceptions et les mêmes erreurs» (Romains, 1964, p. II3). Mais de chevauchement en chevauchement, «la marée finit par monter» et chaque nouvelle génération arrive aux postes de responsabilités dans des conditions légèrement différentes de celles qu’eut à affronter la génération à laquelle elle succède. L’auteur ne se résigne pas tout à fait au déclin de l'idéologie du progrès. Il ne peut s'empêcher d'imaginer la bonne volonté triomphant malgré tout des avanies de l'histoire et il veille à ce que l'œuvre se termine par «une arrière-pensée teintée d'optimisme», même s'il sagit, comme il le dit, «d’un optimisme bien modeste» tel qu'il s'exprime dans le chapitre ultime du dernier volume de l'œuvre, Le 7 octobre, par une phrase laissée à dessein inachevée: «Ce monde moderne serait tout de même quelque chose de bien épatant si ...» (ibid., p. II6-II7).

\section{L'ESPRIT SOCIOLOGIQUE}

L'esprit sociologique qui imprègne la série des Hommes de bonne volonté apparaît de diverses façons, dans les relations dyadiques élémentaires comme dans les phénomènes collectifs. Donnons-en ici quelques échantillons. Il y a tout d'abord les préoccupations de statut que l'auteur prête à différents personnages ou que ceux-ci expriment et qui prennent leur sens dans le cadre de stratégies visant à la manipulation des impressions au sens que Goffman donne à cette expression. L’agent immobilier Haverkamp, par exemple, est très habile à ce jeu. Cherchant à créer un climat de confiance en vue de mettre au point une transaction immobilière délicate, il se heurte de prime abord au scepticisme de ses interlocuteurs. Mais il trouve le ton juste pour le surmonter:

il y avait de la part de Haverkamp un certain ton de voix d'une justesse parfaite ; on ne sait quelles largeur et magnanimité; toute une convention d'élégance morale créée d'un coup et qui, loin d'être démentie ou rendue dérisoire par le caractère des tractations qu'elle recouvrait, n'en prenait que plus de rareté et plus d'éclat. Cette intrusion du chevaleresque dans un ordre de choses où tout n'est d'ordinaire que méfiance, précautions pesées jusqu'à la syllabe et à la virgule, sonnait soudain comme dans une boutique les éperons d'un prince déguisé en marchand (1958 [1936-1942], t. I, p. 693). 
Dans un tout autre genre, le relieur Quinette excelle également à la présentation avantageuse de soi. Tout contact humain est pour lui une interaction permettant d'acquérir un surplus d'influence sur son interlocuteur ou, en l'occurrence, son interlocutrice. Une cliente, entrée dans sa boutique-atelier, ne peut s'empêcher d'être intimidée par son allure et son maintien :

Il avait l'air d'un monsieur, et beaucoup plutôt, malgré certains détails du vêtement, d'un médecin de quartier ou d'un architecte que d'un artisan. Une barbe noire, longue, fournie, d'une coupe assez soignée. Le front dégarni, de cette calvitie nette, à peau fine, qui semble distinguée et studieuse [...] sa voix répondait exactement à sa physionomie. C'était la voix d'un homme de bonne éducation, sans trace d’accent faubourien (ibid., t. I, p. 4I-42).

À un autre niveau de la réalité sociale, l'échange d'influences entre individus est dominé par une pression diffuse du milieu social. Les tranchées de la Première Guerre mondiale constituaient un environnement exerçant une pression particulièrement vive sur l'individu. À certains moments, Jules Romains fait littéralement parler Durkheim par la bouche de ses personnages. Car enfin :

quelle est la force qui est assez grande pour maintenir ces millions d'hommes dans un supplice qui ne finit pas? [...] Quand on y réfléchit bien, il y a une autorité qui domine tout le reste [...] Si l'on s'y réfère, c'est en la déguisant sous des noms d'emprunt $[. .$.$] comme devoir, patriotisme, etc. [...] Or elle porte un$ nom plus franc : c'est la contrainte sociale, tout simplement (ibid., t. III, p. 307).

La lucidité à propos des inégalités sociales est une autre caractéristique de l'œuvre qui postule que toute société, de l'une ou l'autre façon, est stratifiée. Jules Romains fait dire à Jerphanion que

toute société se ramène à un problème de hiérarchie, même quand elle évite de s'en apercevoir, comme la société «libérale » du dix-neuvième siècle ou quand elle se flatte de le supprimer, comme la société socialiste telle que la rêvent certains [...] Si peu qu'il doive s'y établir de hiérarchie, il y aura nécessairement la couche d'en bas, et si raisonnable et apaisante que doive être la distribution des pressions, la couche d'en bas est destinée à les recevoir finalement, à les absorber sans les transmettre (ibid., t. II, p. 610). 
Au cours d'un séjour à Nice, Jallez a noué une relation avec la jeune Antonia, tenancière d'un kiosque à journaux. C'est pour lui l'occasion de réfléchir aux différences de classes: «je n’ai jamais été de ceux qui s’imaginent - par quel naïf orgueil de classe! - que chez un homme du peuple, les mécanismes de pensée sont toujours, et forcément, plus rudimentaires que chez eux. Il y a des hommes du peuple [...] qui sont de naissance des esprits d'une haute distinction» (ibid., t. III, p. 586-587). Pourtant «le degré d'instruction, le genre d'éducation [...] ne sont pas [...] des détails de surface. Même si on le décrète héroïquement, il n'est pas vrai que la condition sociale soit sans aucune importance » (ibid., t. III, p. 598).

Mais Paris reste par excellence l'acteur collectif tout comme le lieu d'observation et d'inspiration de Jules Romains. Même quand il raisonne de façon tout à fait générale, suggérant que toute ville est un système social, c'est l'agglomération parisienne qui lui fournit ses illustrations et ses exemples. La ville - en l'occurrence Paris - est traversée de mouvements collectifs mais ceux-ci ne se déroulent pas de manière désordonnée: ils obéissent à une logique d'ensemble qui a clairement un caractère systémique dans la mesure où elle suggère une distribution spatiale des activités et une séparation du domicile et du lieu de travail, ce qui, à l'époque décrite, est historiquement récent. Le matin, c'est de la périphérie vers le centre qu’ont lieu les déplacements de foule: «Au petit matin, c'est des pentes et des hauteurs de l'Est qu'il en ruisselle le plus : bourgerons, vestes de travail, complets de velours à côtes ; rien que des casquettes » (ibid., t. I, p. I7). Dans le courant de la matinée, l'orientation des déplacements de foule se modifie. «Le gros de la multitude venait non plus de l’Est, mais du Nord-Est de la ville, puis franchement du Nord». Ensuite, «la rotation» se poursuit «du Nord vers le Nord-Ouest et vers l'Ouest» (ibid., t. I, p. I8). Au Sud, se produisent des changements plus ou moins symétriques. Les faubourgs et la périphérie «dardaient plus d’un million d'hommes dans des directions à peu près convergentes » (ibid., t. I, p. I I8-I19). 
Le monde urbain qui fascine Jules Romains est un univers social segmenté. Les rencontres par pur hasard y sont rares car la structuration du système crée des zones de contact et des zones d'évitement. La segmentation du milieu de la grande ville offre une possibilité d'anonymat qui n'existe nulle part ailleurs avec cette force. Dans une petite ville de province,

on trompe sa nostalgie de la grande ville. Et bien! il y a une nostalgie qui est en ce cas la plus difficile à tromper de toutes, une illusion qui est la moins commode à se procurer : celle qu'on va pouvoir se perdre, être n'importe qui, le passant anonyme qui ne rencontrera personne de connu, et que personne ne reconnaîtra, ne remarquera (ibid., t. II, p. I I07).

Se sentir anonyme, c'est déjà un bienfait que toute grande ville vous dispense [...] Mais vous pouvez, en restant inconnu, être remarqué. Jallez éprouvait à Londres une forme supérieure et recuite d'anonymat: être inconnu, mais en outre inaperçu (ibid., t. II, p. I209).

Cette distinction entre la métropole et le bourg provincial rejoint l'opposition entre Gemeinschaft et Gesellschaft proposée par Ferdinand Tönnies, avec cette différence que ce dernier envisage de manière nostalgique le passage de la Gemeinschaft à la Gesellschaft comme un processus de rupture du lien communautaire alors que Jules Romains y voit surtout l'élargissement du domaine de la liberté individuelle, rejoignant ainsi Georg Simmel (2013 [1903]) dont le jugement est néanmoins plus nuancé.

Le cadre de la grande ville - et même de la très grande ville - est toutefois le terrain d'observation rêvé pour qui veut mettre en évidence les contrastes entre les modes de vie dans les différents milieux sociaux. Les heures des repas constituent à cet égard un premier indicateur :

Jadis le repas du soir semblait réglé sur le crépuscule d'hiver. Il s'est rapproché lentement du crépuscule d'été. En 1908, les pauvres se mettent à table un peu avant sept heures; les petits bourgeois à sept heures juste. Une demi-heure plus tard, les familles riches à mœurs tranquilles. En dernier lieu, les gens très affairés, et les gens tout à fait oisifs; ainsi que les convives des dîners de cérémonie et des festins de corps. Mais à huit heures et demie les femmes élégantes sont prêtes pour le théâtre. Les joueurs de manille des cafés de quartiers commencent leur seconde partie. Les rues s'emplissent d'une brève allégresse (1958 [1932-1947], t.I, p. 406). 
Jules Romains a une conscience aiguë de l'importance des normes sociales dans la régulation des comportements. Les normes intériorisées sont les idées que notre milieu social et culturel entretient autour de nous et dont on hésite à s'écarter ouvertement car on sent que les infractions aux normes impliquent toujours un prix à payer, matériellement ou psychiquement. Pour le jeune Wazemmes, alors apprenti dans un atelier de peinture, ce qui se fait ou ce qui ne se fait pas est défini implicitement par l'opinion collective ou, en tout cas, par l'opinion de la majorité. Il croit «qu’au moins en ce qui concerne l’art de vivre, une espèce d'exercice collectif de la raison offre plus de garanties que son exercice individuel». À ses yeux, celui qui s’y connaît le mieux en tout, qui est passé partout, qui sait «les règles » pour chaque cas, et l'opinion qu'il faut avoir, en bien ou en mal, de ce qui nous arrive; celui qui a l'expérience, la sagesse, le discernement, ce n'est pas tel ou tel, c'est «on» (ibid., t. I, p. I7I). Ce «on» est la source de toutes les normes de comportement. Ce qui importe pour Félix Wazemmes, lorsqu’il demande l'avis de quelqu'un, c'est «essayer de deviner quelle est, quelle sera, ou quelle serait, sur tel ou tel point, la pensée de “on” » (ibid).

Maurice Ezzelin reçoit une lettre anonyme l'informant de l'infidélité de son épouse. Qu'est-ce que «on» lui recommande de faire?

Une idée admise veut [...] qu’une lettre anonyme soit méprisable. Un homme de cœur déchire les lettres anonymes et les jette au panier [...] D’autres idées admises, mal conciliables entre elles, viennent compliquer la question. Un mari jaloux est facilement odieux. Mais un mari qui tolère l'inconduite de sa femme est jugé sévèrement. L'expression "l’honneur du mari" reparait souvent en tête d'un feuilleton ou d'un fait divers de journal. Elle a un sens qui ne prête pas à l'équivoque (ibid., t. I, p. III6).

Il y a donc des normes sociales relatives au comportement du mari trompé. Elles indiquent «ce qui se fait» quand on reçoit une lettre anonyme mais elles indiquent également «ce qui se fait» quand la lettre semble être l'avertissement d'une condamnation de l'opinion. 
La sociologie implicite ${ }^{1}$ des Hommes de bonne volonté implique un comparatisme historique non dénué d'un sentiment de nostalgie pour la société d'avant la Première Guerre mondiale. L'instabilité monétaire des années 1920 - phénomène nouveau à l'échelle de l'histoire - est un sujet de conversation récurrent dont s'entretient l'historien retraité Sampeyre avec ses disciples Clanricard et Laulerque: «Largent [...] n'a plus la vertu rassurante qu'il avait autrefois [...] Nous nous demandons malgré nous: “Est-ce que l'an prochain ce que nous gagnons sera encore suffisant?" Et l'idée d'une augmentation possible de traitement ne nous tranquillise pas» (ibid., t. III, p. 677).

La vitesse du changement social est également une source de préoccupations pour ceux qui avaient connu l'époque où la vie se déroulait à un rythme plus lent : «Ce qui importe, c'est d'être sûr du peu qu'on a [...] L’homme est un animal qui s'adapte à presque tout. Mais le travail qu'il fait pour s'adapter, il ne faut pas le lui démolir toutes les cinq minutes ou tous les huit jours » (ibid., t. III, p. 675).

\section{LES CONSÉQUENCES INATTENDUES DES ACTIONS HUMAINES}

Les personnages des Hommes de bonne volonté sont aux prises avec l'imprévisibilité du devenir historique. Alors qu'il a atteint des fonctions ministérielles, Jerphanion ne peut que prendre conscience des conséquences inattendues de toute action organisée.

Souvent ce qui arrive est le contraire de ce qu'on a prévu. Les visiteurs de l'exposition de 1900 prévoyaient un âge pacifique, gorgé de biens matériels; une vie presque trop libre et trop facile; l'épanchement universel de la démocratie et les embrassades d'un socialisme humanitaire. Ils ont eu une guerre mondiale, la famine, la ruine, les dictatures, des socialismes au knout et à la schlague (ibid., t. IV, p. I068).

I L'usage de ce terme n'implique aucune prise de position par rapport aux perspectives de sociologie par la littérature évoquées par David Ledent (2015, p. 8-9). 
Il se laisse alors aller à des propos désabusés qui ne reflètent toutefois qu’une mélancolie passagère: Il existe une vérité de fait, toute simple mais bien gênante [...] À certains moments de l'histoire, il ne reste rien à faire [...] qu'à attendre stoïquement si l'on peut. Ce sont les moments intraitables (ibid.).

Ce paradoxe des conséquences inattendues échappant à l'action rationnelle est aussi le paradoxe de la théorie sociologique, tiraillée entre le rationalisme de l'individualisme méthodologique et les pesanteurs sociales du holisme (Coenen-Huther, 2015).

Jules Romains, homme de lettres, a entretenu avec la sociologie des rapports ambigus qui apparaissent dans les différents aspects de son œuvre et notamment dans sa description de l'univers social segmenté de la grande ville. Sa doctrine littéraire, l'unanimisme, par ses tensions internes, rejoint les travaux de classiques très divers de la sociologie. Cette doctrine hérite d'une tradition déjà ancienne d'émulation entre la littérature et les sciences sociales. Elle entend ouvrir une voie parallèle à celle de l'analyse proprement sociologique qu'il serait d'ailleurs faux d'identifier avec le positivisme de la sociologie durkheimienne.

\section{BIBLIOGRAPHIE}

AGATHON, 191I, L'Esprit de la Nouvelle Sorbonne, Paris, Mercure de France.

BALZAC H. de, 1958, La Comédie humaine, vol. I, Lausanne, Éditions Rencontre.

BOUGLÉ C., 1938 (1935), Bilan de la sociologie française contemporaine, Paris, Alcan.

BOURGET P., 1893, «Introduction», in Répertoire de la Comédie humaine de H. de Balzac, A. Cerfberr et J. Christophe, Paris, Calmann-Lévy.

BOURIN A., 1961, Connaissance de Jules Romains, Paris, Flammarion.

BOUTROUXÉ., 1874, De la contingence des lois de la nature, Paris, Baillière.

CHAZEL F., 2000 (1993), «L'esthétisme sceptique et ses limites en histoire de la sociologie», in Id., Aux fondements de la sociologie, Paris, PUF, p. 85-109. 
-, 1997, «Les ajustements cognitifs dans les mobilisations collectives» in Cognition et sciences sociales, R. Boudon, A. Bouvier et F. Chazel (dir.), Paris, PUF.

COENEN-HUTHER J., 2012, Les Paradoxes de la sociologie, Paris, L'Harmattan.

-, 2015, Quel avenir pour la théorie sociologique? Paris, L'Harmattan.

CUISENIER A., 1969, Jules Romains, l'unanimisme et les hommes de bonne volonté, Paris, Flammarion.

FLAUBERT G., 1980 (1853), Correspondance II (juillet 1851-décembre 1858), Paris, Gallimard, «Bibliothèque de la Pléiade».

HALBWACHS M., 1938, Morphologie sociale, Paris, Armand Colin.

-, 1964, Esquisse d'une psychologie des classes sociales, Paris, Marcel Rivière.

-, 2008, Les Classes sociales, Paris, PUF.

HIRSCHHORN M., 1997, «De la sociologie à la littérature: Durkheim et Jules Romains », in Durkheim d'un siècle à l'autre, Ch.-H. Cuin (dir.), Paris, PUF.

LANCELOT A., 1990a, «Discussion de la communication de Kornel Huvos», Cahiers Jules Romains, 8, p. 82-83.

-, 1990b, «Le personnel politique dans Les Hommes de bonne volonté», Cahiers Jules Romains, 8, p. 159-176.

LASSAVE P., 2002, Sciences sociales et littérature, Paris, PUF.

LE BON G., 1895, Psychologie des foules, Paris, Alcan.

LEDENT D., 2015, «Peut-on parler d'une sociologie implicite du roman?», Revue d'anthropologie des connaissances, 9-3, p. 37I-386.

LEPENIES W., 1990 (1985), Les Trois Cultures. Entre science et littérature, l'avènement de la sociologie, Paris, MSH.

MARX K.et ENGELS F., 1954, Sur la littérature et l'art. Textes choisis, Paris, Éditions sociales.

MAUROIS A., 1965, De Proust à Camus, Paris, Librairie académique Perrin.

ROMAINS J., 1983 (1908), La Vie unanime, Paris, Gallimard.

-, 1976 (1922), Les Copains, Paris, Gallimard. 
-, 1933, Problèmes européens, Petite introduction à l'unanimisme, Paris, Flammarion.

-, 1958 (1932-1947), Les Hommes de bonne volonté, 4 t., Paris, Flammarion.

-, 1964, Ai-je fait ce que j'ai voulu? Paris, Wesmael-Charlier.

RONCAYOLO M., 1990, «Jules Romains et la ville» Cahiers Jules Romains, 8, p.33-56.

RONY O., 1993, Jules Romains ou l'appel au monde, Paris, Laffont.

SIMMEL G., 2013 (1903), Les Grandes Villes et la vie de l'esprit, Paris, Payot et Rivages.

WINOCK M., 1997, Le Siècle des intellectuels, Paris, Éditions du Seuil.

ZOLA É., 1890, Le Roman expérimental, Paris, Éditions Charpentier.

-, 1986, Carnets d'enquête. Une ethnographie inédite de la France, Paris, Plon, «Terre humaine». 